\title{
Tourist information boards on motorways: perceptions, memory effects and decision-making support*
}

\author{
Sven Gross \\ Member of the Institute for Tourism Research, Harz University of Applied Sciences, Wernigerode, Germany
}

Dominik Huber

Research Manager, Outdooractive, Immenstadt, Germany

In recent years more and more tourist information boards have been put up on German motorways. Little research exists with regard to the effects of these information boards. A qualitative research approach was used and 29 semi-structured interviews were conducted to increase knowledge about the relationships between tourist information boards and related tourism behaviour. A model was developed which integrates the elements perception of tourist motorway signage, its memory effects and tourism decision-making processes. Findings suggest that spontaneous driving off the motorway is rarely found and points of interest are more likely to be visited after the trip or used as travel inspiration. This study shows for the first time that tourist information boards can play an important role in tourism decision-making processes and resulting behaviours.

Keywords: tourist signage, information boards, perception, memory, decision making, motorways, tourism, behaviour, Germany

\section{INTRODUCTION}

Billboards and information signs alongside motorways can be found almost everywhere in the world. In recent years, tourist information boards as a specific type of advertisement gained increasing importance in tourism destination marketing. Within the customer journey the inspiration and planning phase is of outstanding importance and tourism marketers aim to intercept customers at relevant contact points, for example travellers driving on a motorway. This conforms with existing literature that suggests that travel-planning processes remain to some extent vague and flexible, which means that spontaneous decision-making can be an important factor for tourism destination marketing (Ballantyne et al. 2008). However, it is still largely unknown how these signboards are noticed by car drivers and their passengers, whether they are remembered and whether they even lead to a spontaneous visit to the designated place.

* Acknowledgements: Many thanks for their support to Saskia Ricken (BA) and Bengt Messner (BA) as well as to the owners and employees of the hotels 'Hotel Zur Post' and 'Hasseröder Ferienpark'. Furthermore, we would like to thank the Research Commission of Harz University, Germany, which enabled this study to be conducted with the aid of its so-called 'research incentive system', all test persons and cooperating companies and institutions (for example, inspektour $\mathrm{GmbH}$, the highest state authority responsible for road traffic). 


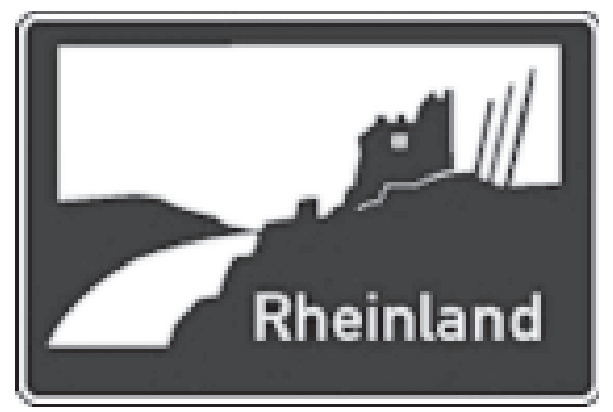

Figure 1 Brown signs on German motorways

In Germany, this signage is regulated according to the 'Guidelines for Touristic Signage' ('RtB2'). Tourist information signs can be used to signpost touristically important destinations on normal roads, touristic routes and motorways (in German: Autobahn; Figure 1) (FGSV 2008: 8ff). Touristic brown signs on motorways have been in use in Germany since 1984; they were introduced in France prior to this date (Steinecke 2013: 96f). These signs denote particularly significant destinations which are either visible from the motorway or are strictly less than $10 \mathrm{~km}$ (as the crow flies) from a motorway junction. These signs typically feature a symbolic representation of touristic attractions or geographical objects (such as cities or landscapes) which are accompanied by a short description (see Figure 1; FGSV 2008: 14).

\section{AIMS AND RESEARCH QUESTIONS}

Up until now, despite extensive research and information by various institutions (for example, the Federal Highway Research Institute, the highest-level traffic authorities, touristic marketing organisations and tourism associations in every German state), there has been no study on how these brown signs affect awareness, memory performance or decision support. For this reason, the aims of the study are to find out to what extent travellers are aware of the touristic brown signs on German motorways, to what extent they stick in the memory, and whether decisions are made as a result of these factors. As stated in the introduction, a trip begins with a form of motorised private transport and generally with a specific destination which should be reached within a specified time period. If, on the way there, a point of interest (PoI) is displayed on one of these touristic brown signs, it can reasonably be assumed that very few people will decide to go there. However the signs inspire the thought that it is possible to go there. Accordingly, one can assume that the touristic brown signs have little to no effect on current behaviour, but rather on future behaviour. However, for this to happen, the brown signs need to stick in a person's memory. Thus, it is necessary to consider the question of whether car drivers notice the signs and can remember PoIs depicted on them. However, because spontaneously exiting the motorway or visiting a destination at a later time can also play a role, this will also be investigated. 


\section{CURRENT STATUS OF RESEARCH}

Signs are important for guiding human behaviour and have therefore been studied from a wide range of disciplinary perspectives. These fields include, for example, geography, psychology, tourism and transport research. In a tourism context signs are used to provide orientation within a destination, for example, in airports and leisure facilities, as well as in hotels and national parks.

When approaching or leaving a destination, the intelligibility of traffic signs is the focus of the studies. The first research on the intelligibility of traffic signs was carried out in 1966 (Zhang/Chan 2013: 1), meaning the discussion is wide-ranging and a host of methodical approaches are used (for example, surveys, eye-tracking, simulators, tests). Contributions which look at general traffic-sign comprehension (for example, Beijer et al. 2004; Färber et al. 2007; Shinar et al. 2003; Zhang/Chan 2013; as well as studies named therein from several countries) can be more commonly found than articles which explicitly place tourists as test objects at the centre of their studies.

Important topics in the general comprehension of traffic signs include factors which influence a person's comprehension, such as age, level of education, driving experience or gender (see for example Zhang/Chan 2013). However, the driver's gaze behaviour (see for example Beijer et al. 2004) as well as their information retention and the style of signposting derived from this (see for example Färber et al. 2007; Gao et al. 2006; Long/Kearns 1996) are the subjects of the studies. In this way it is possible to examine, amongst other things, whether text or symbols are easier to understand on traffic signs; what influence colours have on how the signs are noticed; and how many seconds of reading time are available for signs (overhead or roadside) in order to determine the maximum number of discernible destinations on motorway signs at a particular font size.

Tourism-related studies also cover a wide spectrum of themes, although the foci here are quite different. So, for example, the intelligibility of (traffic) signs is also investigated, including the awareness and intelligibility of street signs among (international) tourists (see for example Dissanayake/Lu 2001; Dudek et al. 1996). However, the question of whether tourists misunderstand and wrongly interpret traffic signs in unfamiliar surroundings (for example, Thailand) or whether they (unconsciously) violate local traffic regulations (Choocharukul/Srioongvikrai 2017: 4518ff) is also investigated. As well as street signs there are also studies in which touristic information signs are evaluated based on their intelligibility - for example, Neill et al. (2015) have five signs for 'tourist information' tested. Furthermore, studies have been carried out on satisfaction with signage (Ballantyne et al. 2008: 30; Findlay/Southwell 2004: 230; Saha 2014: 1), the awareness of street names by tourists, the (potential) correlation with the experience of the destination (Yan/Lee 2014: 432ff), and the significance of the destination in marketing (see for example Bade 2006: 130f; Steinecke 2013: 96f), as well as an observation on the effectiveness of signage in forests (see for example Findlay/Southwell 2004: 230). Further studies deal with the decision-making, information-gathering and planning processes of travellers before and during a trip - and not just for users of motorised personal transport, but also for hikers, cyclists, mountain-bikers, riders, canoeists and kayakers, etc. Other tourismrelated studies which explore signage investigate the (possible) influence on environmental behaviour through signage in a hotel (see for example Goldstein et al. 2008), taking your own litter with you in national parks (see for example Brown et al. 2010: 472ff), or the suggestion of paying a 'voluntary entrance fee' (see for example Steckenreuther/Wolf 2013: 58ff). 
In tourism research there is a series of publications which study decision-making behaviour or elements of it (for example, information-gathering behaviour). This is also reflected in the existing models of purchasing and decision-making behaviour. The decision-making process for holiday trips, particularly the choice of destination, is a focus of tourism research. These studies are not relevant to this study, but rather are primarily studies on the significance of traffic signs for the information-gathering and planning process for trips (see for example Ballantyne et al. 2008: 22ff; Pringle 1999), as well as for spontaneous visits to PoIs (Dahl et al. 2015), so that selected results can be represented. In this way, Pringle (1999) found that driving tourists rarely use traffic signs to make decisions on whether or not to visit a PoI. A study taking the example of Canada, the USA and Norway investigates the extent to which (traffic) signs can persuade motorists to take unplanned routes and/or partake in additional activities in the region - aside from their actual destination (Dahl et al. 2015: 22). According to the authors, more specific results have not yet been published.

To sum up, it can be stated that (touristic) signage has been the subject of academic research for many years, but that up to now there have been practically no academic articles on awareness of them, on (impulsive) decision-making behaviour and on the recall of (touristic) traffic signs among car drivers (Bade 2006: 130).

\section{DEFINITIONAL AND THEORETICAL PRINCIPLES}

The basic unit of behaviour is the purposeful, goal-driven act which is led by 'cognitive processes'. These processes are the thought processes of a person which take place as private, inner incidents (for example, deciding, planning, judging, perceiving). Behaviour thus provides clues and insights into the thoughts and feelings of a person and is often the only access point to their (inner) experience (Gerrig 2018: 4).

The system that communicates between the input stimulus and the behaviour can be understood as an information-processing system. This system receives a stimulus input and processes the incoming information, that is, it transforms and modifies the incoming data to generate an output (Hagendorf 2011: 22). Figure 2 shows some important aspects of this approach. The individual sensory systems process the information from the environment. A processing path then leads directly to the selection of particular actions (for example, grasping). On a different processing path the information is narrowed via the selective function of attention and in the working memory is linked with the knowledge from the person's memory. The resulting representation (for example, object representation of 'strawberries') reaches various cognitive subsystems such as speech (for example, naming) or memory (Hagendorf 2011: 22f).

The current study deals primarily with the visual stimuli of touristic brown signs, how they are noticed (including directed attention), memory performance and decision-making behaviour. For this reason the academic discussion will be presented below insofar as it is relevant to the study at hand.

Awareness can be a response to objects, processes and relationships and is experienced via the sensory organs; in this way, people can see, hear, touch, taste, smell and feel. Awareness is a system of information processing. Using this system, experienced environmental stimuli and inner signals are decoded. In this way they take on a meaning (information content) for the individual and are processed together with other information to form an internal picture of the environment and the self. 


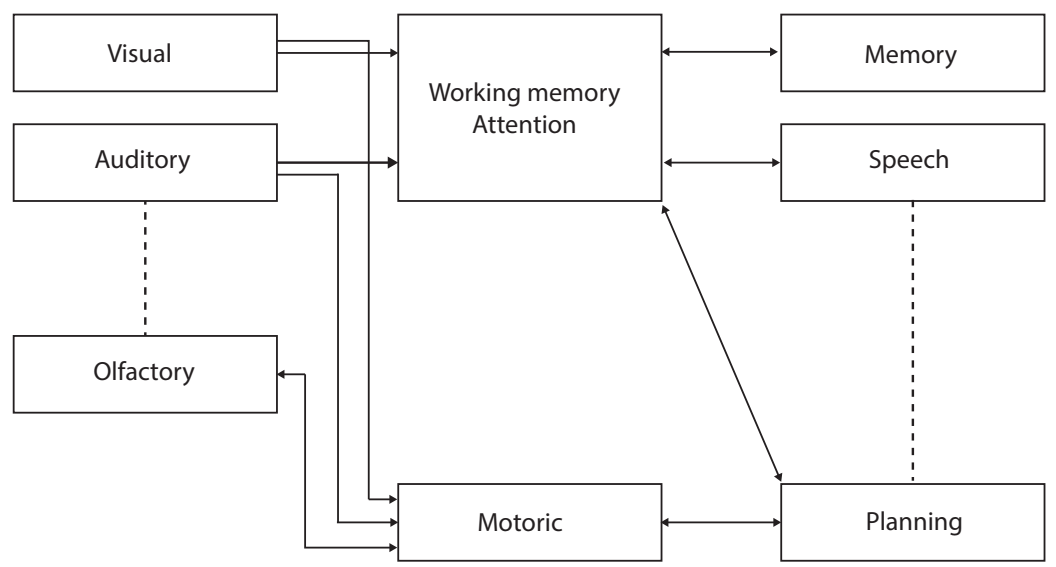

Source: Hagendorf (2011: 22).

\section{Figure 2 Structure of the cognitive system}

Attention directs (selects) awareness so that only stimuli that generate attention are consciously noticed and efficiently processed further (Kroeber-Riel/Gröppel-Klein 2013: 363).

Under the term memory, the information-processing approach refers to the ability of an organism to take in information, to save it and to recall it when required. This form of information processing primarily deals with the flow of information into the memory systems (storage) and out again (Gerrig 2018: 238f).

Currently in psychology, distinctions are made between several memory models, in particular single-store and multi-store models. The first approach postulates an individual memory system where no separate stores are assumed, as in the multi-store model, but, rather, different levels within an individual unit: the long-term memory. Between these levels, alongside automatic processes of encoding (as in the multi-store model), control processes for the processing of information in cognitive performance are assumed (Neath/Surprenant 2005). A widely used model of categorising the memory into various components is the multi-store model by Atkinson/Shiffrin (1968). Here, categorisation takes place according to the time characteristics of the components. This model postulates a sensory storage, a short- and long-term memory. However, this approach is increasingly viewed as outdated (Buchner/Brandt 2017: 402f; Felser 2015: 77; Hofmann/Engelkamp 2017: 117).

Despite this, a structuring of memory into an area of short-term storage and long-term storage has become established, although today the term 'working memory' is preferred when describing the short-term storage of memory (Wentura/ Frings 2013: 119). The long-term memory is in turn distinguished according to different types of saved content. Depending on the author, there are three (Hofmann/ Engelkamp 2017: 5ff), four (Buchner/Brandt 2017: 403; Wentura/Frings 2013: 119) or more types of saved content (Anderson 2013: 160), where the differentiation into declarative and non-declarative memory - regardless of the exact number of memory types - is prevalent (see Figure 3).

On the one side is the storage of acquired structures for the control of physical and linguistic behaviour (procedural memory) and the conceptual structures (semantic memory) derived from these. On the other side is the storage of experienced events 


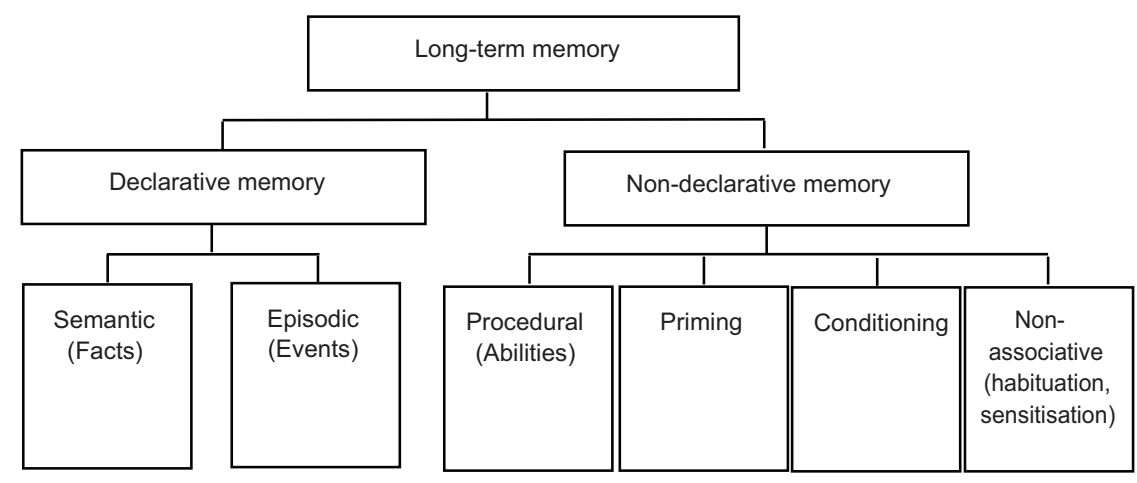

Source: Squire (1987), cited by Anderson (2013: 160).

\section{Figure 3 Structure of the long-term memory}

which are embedded in terms of space and time in the episodic memory (Hofmann/ Engelkamp 2017: 5ff). The procedural memory thus primarily contains the behavioural routines which every person has acquired - knowledge which is extremely difficult to verbalise (for example, riding a bike). The semantic memory contains the knowledge about the world which can be verbalised (for example, general knowledge or definitions). They are valid independently of the person who remembers them (for example, 'A spider always has eight legs') (Anderson 2013: 160; Felser 2015: 79). The episodic memory can ultimately be defined as the memory for place- and time-related personal experiences. This could include parking your car, a holiday or a meeting with friends (Engelkamp 2017: 112). Episodic remembering always involves the special connection to the self, specific phenomenal re-experiencing and phenomenal temporal classification (Tulving 1993; 2005).

Independently of which approach to memory structure is taken, the ability to use information at a later point in time requires the execution of three mental processes: encoding (the first information-processing exercise which leads to a representation in memory), retaining (preservation of encoded information beyond a particular time interval) and recall/memory (retrieval of saved information at a later point in time) (Engelkamp 2017: 115; Gerrig 2018: 258; Wentura/Frings 2013: 102).

As well as awareness and memory, this study also deals with decision. It is a cognitive process of choosing between alternatives, the selection of particular alternatives and the rejection of other options (Gerrig 2018: 367). Decisions in tourism can be made in different ways. Everybody knows decisions which are made in a fraction of a second, but also those that involve weeks and months of preparation. Occasionally a variety of information sources are carefully evaluated, while in other cases decisions are made spontaneously and without any appreciable information basis (Kuß/Tomczak 2007: 107).

According to the traditional perspective of purchasing behaviour, decision-making behaviour can differ according to the extent to which it is cognitively controlled (Decrop 2006; Hyde/Decrop 2011; McCabe et al. 2016; and see Table 1). A distinction is made between decisions with stronger cognitive control (for example, extensive and limited) and decisions with weaker cognitive control (for example, habitual decisions and impulsive decisions) (Kroeber-Riel/Gröppel-Klein 2013: 460). While habitual behaviour affects usual or everyday behaviour, impulsive decisions are 
Table 1 Dominant psychological processes in decision-making behaviour

\begin{tabular}{lccc}
\hline Type of decision & \multicolumn{3}{c}{ Dominant processes } \\
\hline & Emotional & Cognitive & Reactive \\
\cline { 2 - 4 } Extensive & $\mathrm{X}$ & $\mathrm{X}$ & \\
Limited & & $\mathrm{X}$ & $\mathrm{X}$ \\
Habitual & & & $\mathrm{X}$ \\
Impulsive & $\mathrm{X}$ & & \\
\hline
\end{tabular}

Notes: Cognitive: intellectual control of the decision; emotional: activation, primarily of biologically programmed emotions or interpretation of inner arousal; reactive: automatic reaction in action situation.

Source: Weinberg (1994: 174).

characterised by rash action which is unplanned and subject to little intellectual control. These are also called gut decisions or intuitive decisions (see Gigerenzer 2007). Extensive decision-making behaviour describes a comprehensive and, for the most part, conscious problem-solving process. Ultimately, for limited decisions, experiences already exist from which predominantly fixed decision-making criteria result. In a decision-making situation, decision-making criteria no longer need to be developed; rather, one of the available alternatives simply needs to be picked or modified (Kuß/Tomczak 2007: 108).

The current study concentrates on the following theoretical approaches which are used to investigate the research questions. It thus involves visual awareness and episodic memory in the long-term memory. In addition, it is important to note that the memory process takes place in three stages. Behaviour involves cognitive decisions, in particular the impulsive decision to spontaneously exit the motorway due to a touristic brown sign. As well as this, extensive decision-making behaviour (for example, for future plans) and limited decisions (for example, for repeat visits) are also relevant.

\section{METHODOLOGY}

A qualitative research approach was used for the current study. This was chosen to investigate the relatively unresearched field of touristic signage on motorways.

\subsection{Participant selection}

Several studies show that in qualitative research after 12-17 interviews, thematic saturation can be reached. Other authors mention 20-50 interviews as a general recommendation for qualitative research (Francis et al. 2010; Guest et al. 2006; Hagaman/Wutich 2017). These recommendations were used as a benchmark, and thus the aim of the study was to conduct a semi-structured interview and a memory test with 20-30 research participants. Included in the study were both tourists (15 holiday-makers and VFR travellers 'Visiting Friends and Relatives'; UNWTO 2010: $19)$ and commuters (14 students and lecturers) who are regular users of motorised individual transport (that is, car, caravan or motorcycle drivers) on the German motorways and thus also travel to Wernigerode (Germany) by car. 
This study aimed to include tourists and VFR travellers only, but because the recruitment strategy to access respondents in the accommodation facilities and the city centre of Wernigerode was less successful than was hoped for, the target group of respondents was expanded.

The participants were recruited in different ways. Some were asked by hotel employees if they would like to take part in the interview and the memory test (according to a 'Gatekeeper' approach; Creswell 2009: 178). Others were approached directly in their accommodation or in/nearby the university. This provided an arbitrary selection of participants (Schnell et al. 2013: 289). To entice participants to take part, small giveaways were offered (for example, chocolate, postcards) and notices were hung up in the accommodation providers.

Men and women from the age of 17, as well as drivers and front-seat passengers, were included in the study (see Table A1 in Appendix 1) - in Germany, the law allows for accompanied driving from the age of 17, so 17-year-old participants were also included, provided they had the permission of their legal guardian.

\subsection{Survey locations and interview guidelines}

For the interviews, various survey locations in Wernigerode were used: Two accommodation providers; rooms in a university; and private apartments. As well as this, participants were supposed to be recruited at the tourist office and the adjacent market square in Wernigerode. Despite repeated attempts on weekdays and at the weekend, this did not lead to interviews. Contacting other accommodation providers also proved unsuccessful: the contact persons in each case did not respond or did not agree to partake in the study (three rejections).

The interview guide consists of three parts. In the first part, background information (residence, time of arrival) and information about the current trip were gathered. The second part revolved around categories that were deductively derived from the literature analysis and included the themes 'perception of the information boards', 'remembering the information boards', 'decision support through the information boards', 'image building through the information boards' and 'satisfaction with the information boards'. These themes were also used as main categories in the coding process. The third part included socio-demographic information such as age and gender.

\subsection{Pre-test and survey period}

Prior to the data collection, a pre-test was carried out with selected persons (six people). Here, the questionnaire for the semi-structured interview was tested for intelligibility, feasibility and length of time required. As well as this, the participants familiarised themselves with the survey materials used (for example, questionnaires, dictaphone). The materials used were subsequently revised. The survey period was from the beginning of March to the beginning of May 2019. The interviews and memory tests were conducted by one of the authors and two student interviewers, with all participants signing a consent form beforehand. The students were prepared for their tasks as part of a training event which lasted approximately one hour. The interviews lasted between approximately 6 and 18 minutes. 


\subsection{Data processing and analysis}

The data analysis was based on transcribed audio recordings and written notes (memos) which were taken during the interviews. Two student assistants were involved in the transcription process. The qualitative data analysis software 'MaxQDA' was used as a tool for the saving, organisation and encoding of data to simplify the analysis process, whereby a verbatim transcription was made. The thematic analysis (Braun/Clarke 2006) in combination with an approach to qualitative content analysis suggested by Mayring (2002: 114ff; 2015: 65ff) formed the basis of the analysis process (see Figure 4). Based on theoretical considerations, the categories of 'awarenesses', 'memories' and 'behaviour' were derived for further inductive analyses.

The transcriptions were analysed line by line in order to identify and encode themes (inductive approach) which were then assigned to the previously prepared categories. After the analysis of five interviews, the logic of the category formation process was reviewed and the further data analysis was adapted accordingly. To ensure reliability and credibility in the process of qualitative data analysis, this study employed a triangulation approach (Decrop 1999). A second researcher was brought in to review the process of data collection and analysis. Three interviews were analysed by both researchers and the results were compared with each other to confirm their compliance with recognised research practices. To present research results, data-based evidence in the form of textual quotes was translated from German into English.

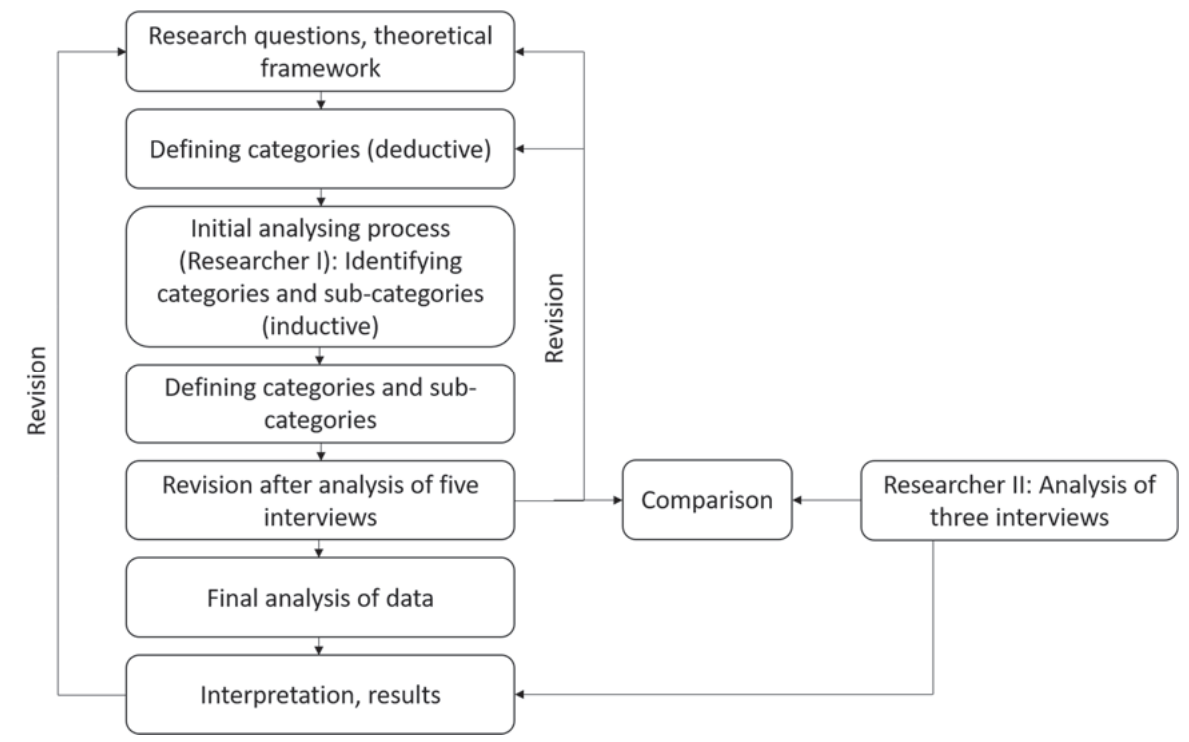

Source: Mayring (2002: 116).

Figure 4 Encoding process and data analysis 
Table 2 Results on awareness and free recall

\begin{tabular}{lccccc}
\hline $\begin{array}{l}\text { Target } \\
\text { group }\end{array}$ & Awareness & Signs free recall & $\begin{array}{l}\text { Target } \\
\text { group }\end{array}$ & Awareness & Signs free recall \\
\hline Commuter & Yes & Yes & Tourist & Yes & Yes \\
Commuter & Yes & Yes & Tourist & Yes & Yes \\
Commuter & Yes & No & Tourist & Rarely & Yes \\
Commuter & Yes & Yes & Tourist & Yes & Yes \\
Commuter & Yes & Yes & Tourist & Yes & Yes \\
Commuter & Yes & Yes & Tourist & Rarely & No \\
Commuter & Yes & Yes & Tourist & Rarely & Yes \\
Commuter & Yes & Yes & Tourist & Yes & Yes \\
Commuter & Rarely & Yes & Tourist & Yes & No \\
Commuter & Yes & Yes & Tourist & Yes & Yes \\
Commuter & No & No & Tourist & Yes & Yes \\
Commuter & Yes & Yes & Tourist & Yes & Yes \\
Commuter & Yes & Yes & Tourist & Yes & Yes \\
Commuter & Yes & Yes & Tourist & Yes & Yes \\
& & & Tourist & Yes & Yes \\
\hline
\end{tabular}

Note: The questions were: 'Do you notice touristic brown signs when you travel by car within Germany?' and 'Do you memorise any of the touristic brown signs or the points of interest listed on them (PoI e.g. landmarks, old town centre, national/natural parks) when you use the German Autobahns? If so, could you give me some examples and name the stretch of Autobahn in question?'.

\section{PRESENTATION OF RESULTS}

It should initially be stated that almost all participants notice the touristic brown signs on the German motorways - only five interviewees explicitly said no, rarely or generally not (see Table 2). The participants were asked to name examples of brown signs (free recall; independent of any specific stretch of motorway). Nearly all the participants were able to give examples of this too. It is interesting that some interviewees, who claimed that they didn't notice the signs or that they noticed them infrequently, were able to give examples. On the other hand, however, two participants who stated that they noticed the signs were not able to give any examples.

\subsection{Awareness}

Below it is outlined whether the interviewees notice the brown signs at all, what the interviewees (first) notice, what they think about the brown signs, and if they discuss them with their fellow passengers. 
Some interviewees explicitly state that they notice the brown signs but quickly forget them again. As well as this, it is stated that awareness of the signs differs according to the situation. Thus, influencing factors are the speed of the vehicle (ID-02, ID-26), the surroundings (ID-22) and whether the interviewee is a driver or passenger in the vehicle, with certain participants more likely to be aware of the signs as a passenger (ID-10, ID-20).

Obviously it always depends on the speed, so when driving a bit faster I just notice them as signs without paying attention to the content. (ID-02)

When I'm driving by myself, I reckon I notice them less than when there's somebody with me. Because then, the odd time, you take a look at the signs that you are passing. But when I'm driving alone, I pay more attention to the blue and yellow signs. (ID-10)

Regarding the awareness of the sign design, it is apparent that there is no homogeneous awareness. However, the majority of the interviewees state that they always see the pictures first. Others see the colour first (brown and white border) or the colour and then the text. Others, in turn, state that they notice the text first or mostly just notice the text. Finally there are people who state that, depending on the sign, they see another aspect first or always notice the entire sign.

\subsection{Memory}

A structuring of memory into an area of short-term storage and long-term storage has become established (see Section 4). As shown, most interviewees were able to name particular brown signs without help. However, it is striking that it is always just a small number of signs that can be named (usually 2-3 signs). In the opinion of the interviewees, these stick in the memory in particular if the tourist attraction in question is visible from the motorway, if the signs or the tourist attractions are particularly notable, if the signs are located in the person's home region, if they are located on regular travel routes, or if the person has more time as a traveller (especially on holiday).

Heichelheim Dumpling World. I just remembered that because it's called the Dumpling World. (ID-09)

They were all some kind of holiday trip or longer car journey to some nice destination or other, so as a result I notice it differently. (ID-02)

Other interviewees state that they cannot name a sign (ID-04, ID-12), that the signs function as brief reminders, but are then quickly forgotten again (ID-04, ID-20) or don't stick in the memory for very long (ID-01, ID-05). Some also said that they could barely remember anything and that the signs just flash by (ID-05, ID-12, ID-23).

Hmmm, well I do notice them. But then after a few seconds I've forgotten them again. Well when you drive past them - sure, you notice these signs, but a short while later they're gone again, to be honest. (ID-04)

For some reason it's just that when you're sitting in your car and some sign or other swishes past, it doesn't seem like proper information. Because there are other signs like 'Take a right in 500 metres', it kind of gets lost a bit. It's not really a source of information that's going to provide cultural education. It's not the same as if I read an article at home about a particular city. You just forget about it again. Sometimes you see it and sometimes you don't. (ID-23) 
The reasons for not remembering or for remembering very little range from a lack of interest (ID-20, ID-24) - and depending on the level of interest the signs stick in the memory to varying extents (ID-22) - to beginner drivers (ID-04), being busy and/or concentrating (ID-12, ID-13) and information overload (ID-23), through to the aspect that the person just wants to get home and is not bothered with these signs (ID-24).

Because I usually travel alone and because I'm actually concentrating on what's happening on the road and not so much on what's going on to the left or the right of the road. (ID-13)

It's more to do with the fact that I want to get to my destination, settle into the hotel and the room. After I arrive, I try to get my bearings and plan what I can do. (ID-24)

\section{3 (Decision-making) behaviour}

As regards behaviour, for some participants absolutely no effects could be noted, while others displayed a range from short to medium-term to long-term behavioural effects.

\subsubsection{Short-term (decision-making) behaviour}

Short-term behaviour includes changing one's driving behaviour, spontaneously exiting the motorway and exchanging views with passengers.

\section{(a) Driving behaviour}

The brown signs do not appear to have negative effects on driving behaviour or road safety. Not a single subject expressed this opinion. Two participants even put forward the theory that the brown signs, rather, have a traffic-calming effect and provide a diversion for drivers (ID-06, ID-07).

Because they ... because they address pleasant, calm topics that make you think and maybe even make you slow down a bit. You might think about a concentration camp or maybe about a nice place where you could spend some time. Even if I just drive at $120 \mathrm{~km} / \mathrm{h}$ instead of 150 $\mathrm{km} / \mathrm{h}$. On the contrary I think that it shortens the motorway, I mean a car journey, by providing diversion and acting as a stimulus to keep your mind fresh. (ID-07)

\section{(b) Exchanging views with passengers}

Most interviewees reported having exchanged views about the brown signs with their fellow passengers - but it is not the rule, rather it is the exception. One person confirmed this without being able to give any examples, others explicitly stated that this 'rarely happens' (ID-01), happens only in 'exceptional cases' (ID-05) or 'may have happened' (ID-20, ID-23). At the other end of the spectrum, a subject stated the following:

In principle I read all signs that I see during a car journey out loud. It's kind of a habit of mine. In keeping with this, I also read out all the brown signs in their entirety. I then incorporate these into a discussion if the opportunity presents itself. (ID-29)

Examples of exchanges with passengers are as follows: Passengers are asked whether they know the PoI in question (ID-01, ID-19), have any information on it (for 
example, ID-19, ID-26) or can give any recommendations (ID-26). It is also reported that drivers let passengers know if they have visited the PoI themselves (ID-03, ID-10) or if a particularly interesting PoI is shown (for example, ID-02, ID-10, ID-20). Finally, it is reported that any children travelling in the vehicle are informed about particular tourist attractions and landscapes (ID-06).

Well, if I know it myself and if I have passengers who I don't know, maybe from BlaBlaCar or another car pool service, then if I'm interested in it myself, I'll ask them if they know anything about it. (ID-09)

And it's kind of a way of educating the kids if you can say 'Now we're in the Teutoburg Forest' - this is where it is. That's something that I find good. (ID-06)

A small number of participants state that there is no exchange of views during the journey, the brown signs generally just pass them by and that neither they nor other people pay them any attention (for example, ID-04, ID-07, ID-24). One subject states that there are no discussions while on the road, but that during breaks they sometimes bring up one or other of the signs (ID-22).

As I said, if these signs register with us, then we shelve the topic for the time being and maybe bring it up during a mid-journey break, or lunch or something like that. (ID-22)

\section{(c) Spontaneously visiting illustrated PoIs}

Even though a variety of behavioural effects can be observed in the majority of people, spontaneously exiting the motorway (that is, an impulsive decision) is rare both with regard to the number of people who have ever done it, and with regard to the frequency of the spontaneous exits. The majority of interviewees state that this has never happened.

I have a kind of travel guide where all of these brown signs and the related tourist attractions are explained for each section of the motorway. It's in my car and I've intentionally and properly used it no more than 2 or 3 times. (ID-05)

Yeah, it was actually at the Bergen-Belsen concentration camp memorial. I said 'Right we have three free hours that we can spend somewhere' or something like that and then we left the motorway and had a look. (ID-26)

The reasons given for not yet having spontaneously left the motorway were as follows: There is a particular destination to be reached and the interviewees want to get there as quickly as possible. This can be the case both for private trips and business trips. Not noticing the signs and a lack of interest in exiting the motorway or taking a break were also mentioned.

No. Definitely not, because I'll only drive there if it's a planned destination. But I wouldn't leave the motorway based on a tourist sign. (ID-12)

From the motorway ... I wouldn't, because we also have a specific destination that we are tied to and we're also limited in terms of time. We really just want to get there so we tend to actually make fewer stops. (ID-15) 


\subsubsection{Medium-term (decision-making) behaviour}

Medium-term behavioural effects might include visiting a PoI on the way home or during a later journey. As assumed (see Section 2), based on a touristic brown sign a PoI is more likely to be visited afterwards on the return journey or during a future journey, that is, deferred.

Yeah, that was exactly the case with Nordhausen. Because it was a nice day and, as I said, it was visible from the motorway. On the journey there I didn't have time. On the return journey ... I remembered it again and then I actually went there with the help of my sat-nav. It was just a short visit, but it was a visit - just to see the place. Because I found it extremely interesting. (ID-02)

That happened to us when my husband was travelling for business. During the last autumn break we just went for a drive along my husband's route and randomly exited the motorway based on one of these signs. (ID-06)

\subsubsection{Long-term (decision-making) behaviour}

Long-term behavioural effects may include effects which influence your life's path, also known as 'life events' in academic literature, as well as the inspiration to visit the depicted PoIs in the future.

\section{(a) Life events}

'Life events' are key incidents which lead to a change in behaviour for a specified period of time (Huber et al. 2017: 30). One interviewee told of an experience with the brown signs which had a considerable influence on her parents' life path. On the way from Dresden to Berlin she saw a sign that resulted in her planning to spend a day at the lake on a subsequent journey to Berlin. She told her parents about the place, and they in turn visited it and ended up moving there.

My parents. And the story continued. They liked it so much that they opened a restaurant there. So the story really is continuing. (ID-04)

\section{(b) (Travel) inspiration for the future}

The signs inspired some participants to make (travel) plans for the future. Be it the compilation of an 'imaginary list' of PoIs (ID-08) that one wants to visit in the future, the idea of a travel route through Germany with the aid of the brown signs (ID-08) or the desire to 'do something like that some time' (for example, ID-15, ID-18, ID-26). As regards the last interviewee mentioned, it seems that while they have addressed the topic, the ultimate step towards making a decision on the trip is yet to be made.

I thought during the drive that when you get older, you can definitely just set off and spend a week visiting places with the help of the signs. Germany is so beautiful and has so much to offer ... . That would actually be a really nice travel route - just heading off without a destination, using the signs as a guide. (ID-06)

A couple of times I actually did think that it's something I ought to do sometime. But the thought that you ought to do something sometime isn't enough in terms of making a concrete 
travel decision. It's kind of the step beforehand. It inspires you a little bit, but doesn't fully motivate you. At least that's how it was for me. (ID-14)

\subsubsection{Further effects on (decision-making) behaviour}

The search for information, a positive attitude towards recommendations, and image formation can cause behavioural changes across all three phases.

\section{(a) Information behaviour}

While the majority of interviewees had never previously looked for information on the signs during or after the journey, some interviewees did do this (at least once).

It's not like you have a lingering feeling that you need to think about it as soon as you get home and then look it up straight away. No. (ID-11)

The information is primarily searched online (Google, Wikipedia, etc.) using a smartphone, tablet or laptop, although people also request information via navigation devices and local or national tourist organisations.

That was at the Wartburg on the way to Weimar. So we actually ... because we wanted some information on the Wartburg that we didn't have to hand, she actually googled it. But she was only able to do this because she was a passenger. (ID-12)

It did happen before. I just remembered where I was driving, and then by the time I drove past the sign I was already thinking 'You can read about it on Wikipedia later'. Just because I was interested in the region. (ID-29)

\section{(b) Recommendation}

Although it is apparent that quite a lot of participants exchange views about the brown signs with fellow passengers, they hardly ever recommend the tourist attractions, cities and/or landscapes shown on the signs after the journey. Just a few interviewees stated that they recommended destinations based on the signs (for example, ID-04, ID-09, ID-24) and that was to their parents, friends or colleagues. Only two interviewees had already been given recommendations by other people (ID-01, ID-27).

\section{(c) Image effects}

Most interviewees state that in their opinion the brown signs that depict landscapes or are used to welcome people to a region such as Franconia, Harz Mountains, Lüneburg Heath, etc., do not have any influence with regard to the image of the destination. Some participants aren't aware of these landscape signs or are of the opinion that they wouldn't elicit any kind of response in them. Others cannot remember any signs of that kind or cannot remember their contents or graphics. Only a small number of interviewees explicitly state that in their opinion these signs have an image effect. As well as this, a certain number of affirmations can be observed, although these are not differentiated, that is, affirmation was given, but not supplemented by any other information ('Definitely has an effect'/‘I think so', for example, ID-06, ID-20, ID-25). 
I think that when you have looked at the sign, you will get a picture. Then you think, 'it was ok or it wasn't okay,' and depending on that, I do think that it contributes to image formation. (ID-27)

More specific statements relate to the repeatedly addressed themes of a sense of home, geographic orientation in Germany and educational purposes. The latter covers both new knowledge for oneself and/or the family, as well as refreshing one's current knowledge.

I come from Lüneburg Heath. So when I drive past the sign I get an immediate sense of home. And then I think 'Ah yes, almost home'. And I get a similar feeling when I'm driving to the Harz Mountains. ... And I feel 'Nearly there now. Lovely ... the mountains'. So straight away I kind of feel at ease. (ID-11)

When I see it, certain aspects that I associate with a region come to mind, i.e. eagles, deer and witches for the Harz mountains. Or heather, heath and sheep for the Lüneburg Heath. Even if all of those things are not on the signs. (ID-16)

\section{DISCUSSION OF RESULTS}

The results of this study have both theoretical and practical implications. The most important finding of this study is that the tourist information boards on German motorways have various effects that influence short-, medium- and long-term behaviour. Based on the theoretical principles and the results achieved, a model was developed (see Figure 5).

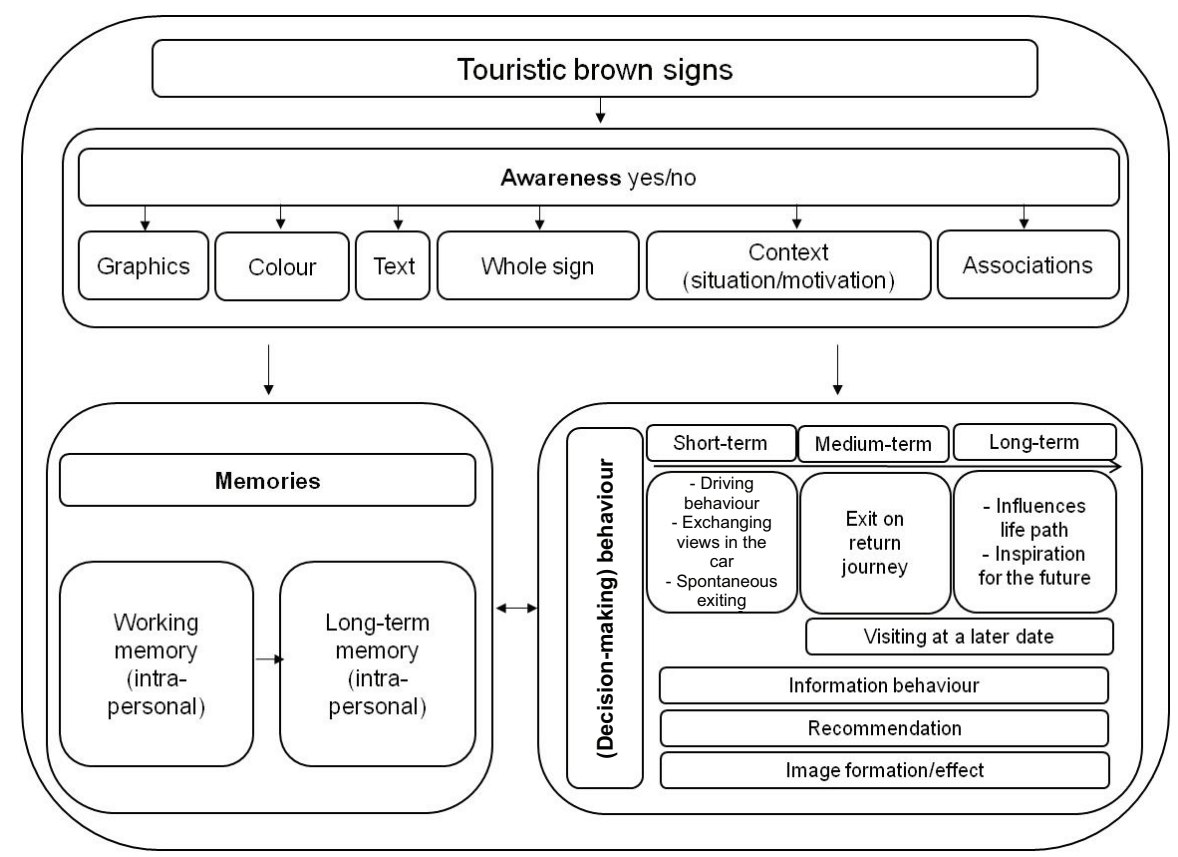

Figure 5 Model for the awareness, memories and behavioural support of brown signs 
The model includes the three elements of the awareness of touristic motorway signage, its admission into long-term and short-term memory and the recipients' decisions based on this. The various elements of the signage design, such as the colours, text, graphics or overall picture have differing impacts on the memory performance of the recipients. Short-, medium- and long-term decision-making behaviour is based both on the retentiveness of the signs and on how the signage is noticed. With the aid of the qualitative interviews, several dimensions of decision-making behaviour were able to be worked out, especially visiting PoI at various time intervals, information-gathering behaviour and behaviour relating to recommendations.

First of all, in terms of awareness and memory, it should be noted that almost all participants are aware of the brown signs and reported that they notice them when they drive. Furthermore, most of the interviewees can also remember at least some of the signs (long-term memory, see Figure 3). Here it can be observed that only a minimal amount of attention is necessary for these effects. For the observed memory effects it cannot be assumed that the people surveyed paid particular attention to the signs or that they intended to memorise the signs when driving past. Rather, they were probably 'encoded' casually, below the attention threshold. This is consistent with a host of results from research into advertising effects, whereby advertising even has an effect if it is casually and unconsciously observed (Felser 2015: 81ff).

It is apparent that the signs grab the attention in different ways - it could be the graphics, the text, the colour, the entire sign, or a combination of the aforementioned aspects that is noticed. However, it is the design that is mentioned most frequently by the participants. Other studies, too, have shown that specific symbols (for example, objects and people with whom one is familiar in the real world) are noticed better than abstract symbols (Zhang/Chan 2013). In the current study, no age- or gender-specific differences were observed in this regard. This, again, is consistent with the results of MacKay/Smith (2006). Their memory study of advertisements demonstrates that age differences can be observed in a typical text-based test format, but not in a situation where framed pictures are the stimuli.

As regards awareness of signs it is apparent that a distinction can be made between emotional and cognitive effects. On the one hand, knowledge is communicated or acquired with the aid of the brown signs, and on the other hand they trigger feelings such as feelings of security (for example, landmarks when heading off on holiday) and a sense of home - firstly in one's home region and secondly for repeat visitors in particular, when headed towards their intended destination. The significance of emotional appeal in touristic marketing and its influence on future travel behaviour (consumption vision) was pointed out by Walters et al. (2012). In their study on future travel intentions and emotions and their effects on touristic consumer behaviour, the authors note that 'an elaborate and quality consumption vision, mediated by an emotional response, can significantly influence the tourism consumers' interest in an advertised product to the point where they are likely to seek closure on their decision and make an immediate purchase' (Walters et al. 2012: 380).

Here, it is also important to mention that a discussion takes place in the car relatively often (for example, discussions about PoI, looking for information during the journey), meaning that more pronounced memory and behavioural effects brought on by the signs are to be expected. Due to these exchanges of views it is also to be expected that decisions on visiting a PoI (spontaneously or later) are often not made alone. This question was not explicitly investigated in this study, however there are several studies that look into the important role of (marriage) partners and family members (Bronner/de Hoog 2008; Kozak 2010). The question of how the decision is 
reached about whether to exit the motorway spontaneously or at a later time should also be investigated with regard to this question in future studies.

Even though a variety of behavioural effects can be observed in the majority of people, spontaneously exiting the motorway (that is, an impulsive decision) is rare. This correlates with the studies by Pringle (1999) and Saha (2014), which found that visitors rarely rely on signage during a journey to make decisions about attractions, activities and places to visit. As assumed, based on a touristic brown sign a PoI is more likely to be visited afterwards on the return journey, during a future journey, or used as inspiration. In the rare case of an impulsive decision due to the effect of information boards, the signs themselves are not always the sole catalyst for a trip, rather they are one of many factors. This finding, along with effects that influence one's life path, can be demonstrated for the first time as far as the authors are concerned.

Finally, it is shown that from the interviewees' point of view, the touristic brown signs have little or no negative effects on driving behaviour. Some participants even see positive effects on road safety in terms of traffic calming. The possible effects of this kind of signage have already been pointed out by Färber et al. (2007: 43ff).

A number of practical recommendations can be derived from the information presented thus far. The participants notice the brown signs, can remember them, and they lead to (decision-making) behavioural effects. If, at the place where they make their decisions (for example, when at home and deciding where to go using the internet, brochures, etc.), the tourists encounter exactly the same motifs (be they designs, drawings or pictures) as they have previously seen, it can be assumed for the most part that they will remember these motifs. This can be inferred, even if the results are interpreted cautiously. Here it can be observed that only a minimal amount of attention is necessary for these effects. For the observed memory effects it cannot be assumed that the people surveyed paid particular attention to the signs or that they intended to memorise the signs when driving past. Rather, they were probably 'encoded' casually, below the attention threshold. This is consistent with a host of results from research into advertising effects, whereby advertising even has an effect if it is casually and unconsciously observed (Felser 2015: 81ff). For this reason, the responsible authorities, such as destination marketing organisations, city, county and/or regional councils, touristic service providers such as accommodation and transport companies, should integrate the designs on the brown signs into their marketing materials. If one is to give any credence to the results of the studies, repeated exposure to the designs, texts, etc., on the brown signs should lead to a positive appraisal. This is termed the 'mere exposure effect'. The repeated exposure alone of an individual to a stimulus is a sufficient condition for the improvement of that individual's attitude towards the stimulus (Zajonc 1968: 1).

In a step towards digitisation, the brown signs could be combined with technological components. This would make it possible to develop a website and/or app with information, photos, links, etc., on the individual tourist attractions, cities and landscapes. An app could also employ beacon technology so that when driving past a sign - with the appropriate setting on a smartphone - further information on the PoI in question could be provided. This could be playfully turned into a quiz app for the passengers so that the driver of the car isn't distracted, but the passengers can discuss the PoI. However, GPS-based reward-points schemes involving a specified number of signs and the associated rewards could make the currently static signs into an interactive tool - in hiking regions there are already similar offers (Lück/Gross 2016.) As well as this, the participants repeatedly expressed their desire for a revised design 
(for example, different colour, more unique style), provision of distance (km information) and the exact exit, as well as dual-language signs (German and English). The responsible authorities should also give thought to this.

\section{CONCLUSION, LIMITATIONS AND FUTURE PROSPECTS}

It can be noted that (practically) all participants notice the touristic brown signs, meaning the function of the instruction provided by these signs can be ascertained in effect. Furthermore, free recall can be observed in almost all participants, while the majority also displayed short-, medium- or long-term behavioural effects.

Even when the interviews are approached with care and observe established standards of academic work, this study is not without limitations. Firstly, it should be noted that only Germans were included in the study, the focus was on a specific destination, and participants were chosen by means of random selection. A further limitation is that the process of remembering is a reconstructive process, meaning that the described memories usually do not exactly correspond to the original experiences. In this context, the difficulties of recruiting participants should also be pointed out. This can be qualified by the fact that only 29 interviewees were able to be included in the study. It is therefore not possible to draw any generalisations from the results, although these results can be applied to other contexts. On a positive note, it can also be pointed out that investigation of the subjective attitudes, awarenesses, memories and behavioural effects could be seen as a strength of this research.

Finally, the quality criteria for an analysis of contents are referred to. As with quantitative studies, these are the measure of its reliability and validity. To determine the reliability of the content analysis, as a rule all of the analysis or (relevant) excerpts are carried out by several people and the results are then compared (intercoder reliability). Using this process, objectivity is actually measured as the independence of the results from the person carrying out the investigation (Mayring 2015: 124). Therefore, in order to meet the quality criteria of the present study, the analysis of relevant excerpts was carried out by both authors.

For future research projects the use of a bigger sample size and the inclusion of participants from different countries are recommended to allow more reliable statements to be made for the purposes of the applicability of statistical tests. As well as this, individual elements of the study (for example, gaze behaviour, design, image effects) could be researched in more depth in future studies. Gender and age differences and behavioural variations between drivers and co-drivers or tourists and commuters were not part of this exploratory study. Future studies in the form of quantitative research designs may investigate these dimensions and their relationships with the effects of tourist information boards.

\section{REFERENCES}

Anderson, J.R. (2013): Kognitive Psychologie, 7th edn, Berlin: Springer VS.

Atkinson, R.C., Shiffrin, R.M. (1968): Human memory: a proposed system and its control processes, in: Spence, K.W., Spence, J.T. (eds), The Psychology of Learning and Motivation: Advances in Research and Theory, Vol. 2, New York: Academic Press, 89-195.

Bade, M. (2006): Marketing entlang der Autobahn, in: Heinritz, G., Lentz, S., Tzschaschel, S. (eds), Nationalatlas Deutschland - Leben in Deutschland (Band 12), Leipzig: Springer Spektrum, 130-131. 
Ballantyne, R., Hughes, K., Weiler, B., Moscardo, G. (2008): Tourists'Use of Roadside Signage: A Case Study of the Great Southern Route, Gold Coast, Queensland: Cooperative Research Centre for Sustainable Tourism.

Beijer, D., Smiley, A., Eizenmann, M. (2004): Observed driver glance behaviour at roadside advertising signs, in: Transportation Research Record: Journal of the Transportation Research Board, 1899(1), 96-103.

Braun, V., Clarke, V. (2006): Using thematic analysis in psychology, in: Qualitative Research in Psychology, 3(2), 77-101.

Bronner, F., de Hoog, R. (2008): Agreement and disagreement in family vacation decisionmaking, in: Tourism Management, 29(5), 967-979.

Brown, T.J., Ham, S.H., Hughes, M. (2010): Picking up litter: an application of theory-based communication to influence tourist behaviour in protected areas, in: Journal of Sustainable Tourism, 18(7), 879-900.

Buchner, A., Brandt, M. (2017): Gedächtniskonzeptionen und Wissensrepräsentationen, in: Müsseler, J., Rieger, M. (eds), Allgemeine Psychologie, 3rd edn, Berlin: Springer, 401-434.

Choocharukul, K., Srioongvikrai, K. (2017): Road safety awareness and comprehension of road signs from international tourists' perspectives: a case study of Thailand, in: Transportation Research Procedia, 25, 4518-4528.

Creswell, J.W. (2009): Research Design: Qualitative, Quantitative, and Mixed Methods Approaches, Thousands Oaks, CA: SAGE Publishing.

Dahl, T.I., Dahl, L., Dalbakk, J.-A., Langseth-Eide, B.E. (2015): The sign language of road trips: guides to authentic cultural learning and understanding?, in: Proceedings of the International Adventure Conference 2015, Sheffield: Sheffield Hallam University, 21-22.

Decrop, A. (1999): Triangulation in qualitative tourism research, in: Tourism Management, 20(1), 157-161.

Decrop, A. (2006): Vacation Decision Making, Wallingford: CABI.

Dissanayake, S., Lu, J.J. (2001): Traffic control device comprehension: differences between domestic and international drivers in USA, in: IATSS Research, 25(2), 80-87.

Dudek, C.L., Huchingon, R.D., Trout, N., Chester, D. (1996): International tourist guidance needs and understanding of selected guide signs in Florida, in: Transportation Research Record, 1550(1), 37-47.

Engelkamp, J. (2017): Einleitung zum episodischen Gedächtnis, in: Hofmann, J., Engelkamp, J. (eds), Lern- und Gedächtnispsychologie, 2nd edn, Berlin: Springer, 111-117.

Färber, B., Färber, B., Siegener, W., Süther, B. (2007): Aufnahme von Wegweisungsinformationen im Straßenverkehr - AwewiS, Bremerhaven: Wissenschaftsverlag NW.

Felser, G. (2015): Werbe- und Konsumentenpsychologie, 4th edn, Berlin: Springer.

FGSV (Forschungsgesellschaft Straßen- und Verkehrswesen) (2008): Richtlinien für die touristische Beschilderung (RtB), Köln: FGSV Verlag.

Findlay, C., Southwell, K. (2004): I just followed my nose: understanding visitor wayfinding and information needs at forest recreation sites, in: Managing Leisure, 9(4), 227-240.

Francis, J.J., Johnston, M., Robertson, C., Glidewell, L., Entwistle, V., Eccles, M.P., Grimshaw, J.M. (2010): What is an adequate sample size? Operationalising data saturation for theorybased interview studies, in: Psychology and Health, 25(10), 1229-1245.

Gao, X.W., Podladchikova, L., Shaposhnikov, D., Hong, K., Shevtsova, N. (2006): Recognition of traffic signs based on their colour and shape features extracted using human vision models, in: Journal of Visual Communication and Image Representation, 17(4), 675-685.

Gerrig, R.J. (2018): Psychologie, 21st edn, Hallbergmoos, Germany: Pearson.

Gigerenzer, G. (2007): Gut Feelings: The Intelligence of the Unconscious, New York: Viking.

Goldstein, N.J., Cialdini, R.B., Griskevicius, V. (2008): A room with a viewpoint: using social norms to motivate environmental conservation in hotels, in: Journal of Consumer Research, 35(3), 472-482.

Guest, G., Brown, A., Johnson, L. (2006): How many interviews are enough? An experiment with data saturation and variability, in: Field Methods, 18(1), 59-82. 
Hagaman, A.K., Wutich, A. (2017): How many interviews are enough to identify metathemes in multisited and cross-cultural research? Another perspective on Guest, Bunce, and Johnson's (2006) landmark study, in: Field Methods, 29(1), 23-41.

Hagendorf, H. (2011): Wahrnehmung, in: Hagendorf, H., Krummenacher, J., Müller, H.-J., Schubert, T. (eds), Wahrnehmung und Aufmerksamkeit: Allgemeine Psychologie für Bachelor, Berlin: Springer, 13-30.

Hofmann, J., Engelkamp, J. (2017): Kapitelübersicht, in: Hofmann, J., Engelkamp, J. (eds), Lern- und Gedächtnispsychologie, 2nd edn, Berlin: Springer, 1-6.

Huber, D., Milne, S., Hyde, K.F. (2017): Biographical research methods and their use in the study of senior tourism, in: International Journal of Tourism Research, 19(01), 27-37.

Hyde, K.F., Decrop, A. (2011): New perspectives on vacation decision making, in: International Journal of Culture, Tourism and Hospitality Research, 5(2), 103-111.

Kozak, M. (2010): Holiday taking decisions: the role of spouses, in: Tourism Management, 31(4), 489-494.

Kroeber-Riel, W., Gröppel-Klein, A. (2013): Konsumentenverhalten, 10th edn, München: Verlag Vahlen.

Kuß, A., Tomczak, T. (2007): Käuferverhalten: Eine marketingorientierte Einführung, 4th edn, Stuttgart: UTB.

Long, G.M., Kearns, D.F. (1996): Visibility of text and icon highway signs under dynamic viewing conditions, in: Human Factors, 38(4), 690-701.

Lück, M., Gross, S. (2016): Stamp books in the Harz mountains, Germany: fun not just for children, in: Richins, H., Hull, J. (eds), Mountain Tourism: Experiences, Communities, Environments and Sustainable Futures, Wallingford, UK: CABI, 58-66.

MacKay, K.J., Smith, M.C. (2006): Destination advertising: age and format effects on memory, in: Annals of Tourism Research, 33(1), 7-24.

Mayring, P. (2002): Qualitative Sozialforschung, Weinheim, Germany: Beltz Verlag.

Mayring, P. (2015): Qualitative Inhaltsanalyse: Grundlagen und Techniken, 12th edn, Weinheim: Beltz Verlag.

McCabe, S., Li, C., Chen, Z. (2016): Time for a radical reappraisal of tourist decision making? Toward a new conceptual model, in: Journal of Travel Research, 55(1), 3-15.

Neath, I., Surprenant, A.M. (2005): Mechanisms of memory, in: Lamberts, K., Goldstone, R.L. (eds), Handbook of Cognition, London: SAGE Publishing, 221-238.

Neill, J.M., Hurwitz, D.S., Olsen, M.J. (2016): Alternative information signs: evaluation of driver comprehension and visual attention, in: Journal of Transportation Engineering, 142(1), $1-12$.

Pringle, N. (1999): Road Travellers Study: Research Report Prepared for Tourism Queensland, Brisbane: Yann, Campbell, Hoare \& Wheeler.

Saha, T. (2014): Visitors' acuity of tourism signs in Australia, in: Proceedings of the 26th ARRB Conference, Sydney, New Wales, Australia, 7(4), 1-9.

Schnell, R., Hill, P.B., Esser, E. (2013): Methoden der empirischen Sozialforschung, 10th edn, München: Oldenbourg.

Shinar, D., Dewar, R.E., Summala, H., Zakowska, L. (2003): Traffic sign symbol comprehension: a cross-cultural study, in: Ergonomics, 46(15), 1549-1565.

Squire, L.R. (1987): Memory and Brain, New York: Oxford University Press.

Steckenreuter, A., Wolf, I.D. (2013): How to use persuasive communication to encourage visitors to pay park user fees, in: Tourism Management, 37(August), 58-70.

Steinecke, A. (2013): Management und Marketing im Kulturtourismus: Basiswissen - Praxisbeispiele - Checklisten, Wiesbaden, Germany: Springer VS.

Tulving, E. (1993): What is episodic memory?, in: Current Directions in Psychological Science, 2(3), 67-70.

Tulving, E. (2005): Episodic memory and autonoesis: uniquely human?, in: Terrace, H.S., Metcalfe, J. (eds), The Missing Link in Cognition: Origins of Self-Reflective Consciousness, New York: Oxford University Press, 3-56.

UNWTO (United Nations World Tourism Organization) (2010): International Recommendations for Tourism Statistics 2008, New York: UNWTO. 
Walters, G., Sparks, B., Herington, C. (2012): The impact of consumption vision and emotion on the tourism consumer's decision behaviour, in: Journal of Hospitality \& Tourism Research, 36(3), 366-389.

Weinberg, P. (1994): Emotionale Aspekte des Entscheidungsverhaltens: Ein Vergleich von Erklärungskonzepten, in: Forschungsgruppe Konsum und Verhalten (ed.), Konsumentenforschung, München: Vahlen, 171-181.

Wentura, D., Frings, C. (2013): Kognitive Psychologie, Wiesbaden, Germany: Springer VS.

Yan, L., Lee, Y.M. (2014): Tourist perceptions of the multi-linguistic landscape in Macau, in: Journal of China Tourism Research, 10(4), 432-447.

Zajonc, R.B. (1968): Attitudinal effects of mere exposure, in: Journal of Personality and Social Psychology Monographs, 9(2), 1-27.

Zhang, T., Chan, A.H.S. (2013): Traffic sign comprehension: a review of influential factors and future directions for research, in: Proceedings of the International Multi Conference of Engineers and Computer Scientists Vol. II, Hong Kong: IMECS. 
50 Journal of Qualitative Research in Tourism, Vol. 1 No. 1

\section{APPENDIX 1}

Table A1 Researchparticipant characteristics

\begin{tabular}{|c|c|c|c|c|c|}
\hline ID & $\begin{array}{l}\text { Driver or } \\
\text { passenger }\end{array}$ & Gender & Type & Age & $\begin{array}{l}\text { Kilometres travelled } \\
\text { per year }\end{array}$ \\
\hline 01 & Driver & Male & Tourist (VFR traveller) & 17 & $15-20000$ \\
\hline 02 & Driver & Male & Commuter (Student) & 25 & $35-40000$ \\
\hline 03 & Driver & Female & Commuter (Student) & 23 & $12-13000$ \\
\hline 04 & Passenger & Female & Commuter (Student) & 24 & $>10000 \mathrm{~km}$ \\
\hline 05 & Driver & Male & Commuter (Lecturer) & 61 & $23000 \mathrm{~km}$ \\
\hline 06 & Driver & Female & Commuter (Lecturer) & 34 & $>10000 \mathrm{~km}$ \\
\hline 07 & Driver & Male & Commuter (Lecturer) & 47 & $<5000 \mathrm{~km}$ \\
\hline 08 & Driver & Female & Commuter (Student) & 22 & $12000 \mathrm{~km}$ \\
\hline 09 & Driver & Male & Commuter (Student) & 21 & $15000 \mathrm{~km}$ \\
\hline 10 & Passenger & Female & Commuter (Student) & 23 & $5-7000 \mathrm{~km}$ \\
\hline 11 & Driver & Female & Commuter (Student) & 22 & approx. $15000 \mathrm{~km}$ \\
\hline 12 & Driver & Male & Commuter (Lecturer) & 60 & approx. $20000 \mathrm{~km}$ \\
\hline 13 & Driver & Female & Commuter (Lecturer) & 43 & approx. $30000 \mathrm{~km}$ \\
\hline 14 & Driver & Male & Commuter (Lecturer) & 48 & approx. $40000 \mathrm{~km}$ \\
\hline 15 & Driver & Female & Commuter (Lecturer) & 44 & approx. $20000 \mathrm{~km}$ \\
\hline 16 & Driver & Male & Tourist (Holidaymaker) & 57 & approx. $25000 \mathrm{~km}$ \\
\hline 17 & Driver & Male & Tourist (Holidaymaker) & 38 & approx. $20000 \mathrm{~km}$ \\
\hline 18 & Driver & Male & Tourist (Holidaymaker) & 67 & $\begin{array}{c}\text { approx. 15-20 } 000 \\
\mathrm{~km}\end{array}$ \\
\hline 19 & Passenger & Female & Tourist (VFR traveller) & 30 & approx. $5000 \mathrm{~km}$ \\
\hline 20 & Driver & Male & Tourist (VFR traveller) & 28 & approx. $15000 \mathrm{~km}$ \\
\hline 21 & Driver & Male & Tourist (VFR traveller) & 57 & approx. $25000 \mathrm{~km}$ \\
\hline 22 & Driver & Male & Tourist (Holidaymaker) & 50 & $15-20000 \mathrm{~km}$ \\
\hline 23 & Driver & Female & Tourist (Holidaymaker) & 26 & $5000-6000 \mathrm{~km}$ \\
\hline 24 & Passenger & Female & Tourist (Holidaymaker) & 31 & $45000 \mathrm{~km}$ \\
\hline 25 & Passenger & Female & Tourist (Holidaymaker) & 60 & $\mathrm{n} / \mathrm{s}$ \\
\hline 26 & Driver & Male & Tourist (Holidaymaker) & 36 & $70000 \mathrm{~km}$ \\
\hline 27 & Passenger & Female & Tourist (Holidaymaker) & 65 & approx. $10000 \mathrm{~km}$ \\
\hline 28 & Driver & Male & Tourist (VFR traveller) & 26 & $16000 \mathrm{~km}$ \\
\hline 29 & Driver & Male & Tourist (VFR traveller) & 22 & $10-20000 \mathrm{~km}$ \\
\hline
\end{tabular}

